\title{
WIRELESS SENSOR AND ACTOR IN UNDERWATER COMMUNICATION
}

\author{
Abhijeet Ashish*1, Pankaj Kumar ${ }^{2}$
}

*Corresponding Author: -

\begin{abstract}
: -
This paper represents the wireless communication system in underwater and also as well as underground system. the wireless sensor and actor work for this. The main motive for this paper is to gives the world a real communication to find any systems, obstacles, the wireless sensor sense the function which is happened in the water, and also helpful in underground. Wireless sensor \& actor works on distributing sensing and acting task. The sensor collects the information from the real world and the actor perform the task to solve the communication process. this paper is very useful in the communication system in underwater.
\end{abstract}

Keywords: sensor and actor, software, battery, hydrophone 


\section{INTRODUCTION:}

In the modern time the growth in wireless networks is so rapid. Wireless communication continues to enjoy this growth in the cellular telephony, wireless internet and wireless home networking. With Wireless LAN (WLAN) technology, computer networks could achieve connectivity with a useable amount of bandwidth without being networked via a wall socket. New generations of handheld devices allowed users access to stored data even when they travel, Recent technological advances have lead to the emergence of distributed wireless sensor and actor networks which are capable of observing real world, processing the data, making

decisions based on the observations and performing appropriate actions. These networks can be an integral part of systems such as battlefield surveillance and microclimate control in buildings, nuclear, biological and chemical attack and the senor also work inside the water this sensor is helpful to the human because the main motive of this sensor is to find the resources and also find any precious aura also predict what's going in the water. For example, in the case of a fire, sensors relay the exact origin and intensity of the fire to water sprinkler actors so that the fire can easily be extinguished before it becomes uncontrollable. We believe that an in-depth understanding of how to communicate underwater is a crucial step in the development, civil use, and protection of the oceans, as well as the safety and growth of dive professionals. Some divers insist on principle that the silence of the deep is one of the most appealing features of this sport, and that taking any form of communication on a dive in water. Many protocols and algorithms have been proposed for Wireless sensor in recent year

People who work underwater must utilize communications with their partners and the surface for both logistical support and safety. In WSANs, the phenomena of sensing and acting are performed by sensor and actor nodes.

\section{Architecture of WSA:}

In WSA, the roles of sensor and actor nodes are to collect data from the environment and perform appropriate actions based on this collected

data, respectively. these nodes are scattered in the sensor/actor field while the sink monitors the overall network and communicates with the task manager node and sensor/actor nodes.in this architecture we use the sensor which sense the data which is coming from the task manager and the sink will send data to the sensor actor field this field is created a function which gives us idea to happen something in underwater. The area where something happen is known as the event and other is known as sink. Sensors detecting a phenomenon either transmit their readings to the actor nodes which process all incoming data and initiate appropriate actions, or route data back to the sink which may issue action commands to actors. We call the real case as the Architecture due to the non-existence of central controller. The most important characteristic of sensor actor communication is to provide low communication delay due to the proximity of sensors and actor. in WSA multiple actors can receive the information from sensors about the sensed phenomenon. The sensor work in the water ,this sense any material available in the water and send a signal to the actor the actor collect the information and sink it and then send it to the real destination.

\section{coordination among actors :}

in WSA actors communicate with each other in addition to communicating with sensors. Actor- actor communication occur in the following situations:

- The actor receiving sensor data may not act on the event area due to small action range or insufficient energy.

- One actor may not be enough to perform the required action, thus, other nearby actors should be triggered.

\section{COMMUNICATON UNDER WATER:}

When the divers dive into water for some mission in a group then it is so tough to communicate to their partners in this case they use the sensors which be used underwater. And we also establish communication between our partners who is on the ground and also who is in the under water. The sensor helps us to find the driver in the water what is their exact position. There are different type of communication are in under water.

\section{Line-pull communications:}

Line-pulls is one of the oldest methods used to communicate between divers and surface. A diver descends pulling along a line from the boat that stretches through the water. To communicate, either end uses a series of one, two, three or four tugs - agreed upon before the dive and depending on the dive itself - and in general a long tug means "I'm about to speak", and an equal

pull in response means "I'm listening". It seems primitive, and it is, but it is still widely used by people working who only need to communicate occasionally 


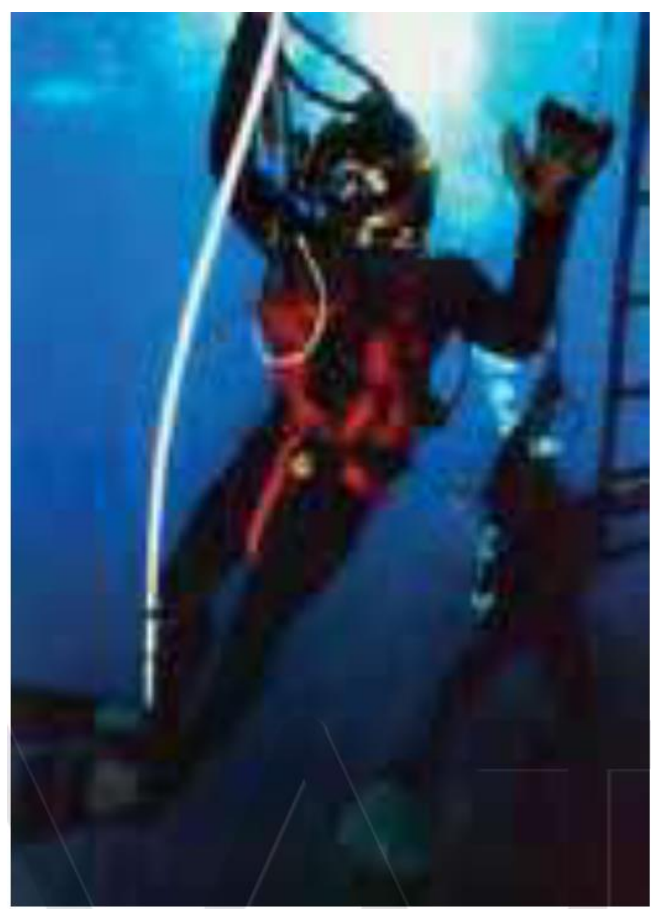

In this figure the diver connected with a line and if he has to require to establish a connection he speak I am listening that is pull communication.

Another form of communication that can be compared to sign language and Morse code : basic acoustic signallers that generate audible noises underwater. We also use the Bluetooth device to make a communication , with the help of communication device we establish a connection in near thing and person we also implement a chip in the wireless device

two things are required in order to speak underwater: a sufficiently large volume of air in front of the mouth, and the facial mobility needed to speak as we do on the surface, for the communication system we need power so we use battery for this the cell in put in the device .which is given to the diving person .and also put it inside the water to seach any precious things and also implement a camera in it to search the material and the person.

\section{HYDROPHONE:}

The HYDROPHONE is very important device to establish a communication under water. Hydrophones are waterproof loudspeakers that use more or less elevated frequencies and full protection from the water to transmit sound waves in the audible band underwater. They are often used to broadcast music in the pool, and less frequently for classic dive communications. hydrophones are used in fishing to attract specific

types of fish depending on the frequency used.

\section{SECURITY:}

Security is a big concern in wireless networking, especially in m-commerce applications . Mobility of users increases the security concerns in a wireless network. Current wireless networks employ authentication and data encryption techniques on the air interface to provide security to its users. The security is also provided by the sensor and the chip which trace the material and the thing which is available in the connection of the actor. The chip is very helpful in the security purpose.

\section{CONCLUSION \& FUTURE WORK:}

This paper identifies and describes the various research issues and challenges available in the wireless sensor and actor function. We fisrt presented the wireless sensor and actor and the work of the sensor in underwater .the sensor sense the diving person and it also sense the precious thing which is available in the water . the wireless sensor and actor is very useful in the water, I think our world will be taken so benefits. when the person goes to the water the sensor detect the person and the data will be fetch through the actor and send a message to the person which is available on the earth.

\section{REFERENCES:}

[1].K. Akkaya, M. Younis, A survey on routing protocols for wireless sensor networks, AdHoc Networks, in press, 10.1016/j.adhoc.2003.09.010

[2]. OCAEN GROUP www.oceanreefgroup.com

[3].M. Haenggi, Mobile sensor-actuator networks: opportunities and challenges, in: Proc. 7th IEEE Int. Workshop, Frankfurt, Germany, July 2002, pp. 283-290 